\title{
Google Android Operating System: A Review
}

\author{
Palak Khanna \\ Assisstant Professor \\ Department of Computer Application \\ CGC Landran,Chandigarh
}

\author{
Amandeep Singh \\ Research Scholar \\ Guru Kashi University \\ Talwandi Sabbo,Bathinda
}

\begin{abstract}
Nowadays, as the developing of hardware for cellular phones is becoming much better, the performance index is significantly higher than the legitimate specifications of the software configuration. Features of Mobile phone's depends completely on software. As the Android operating system is getting considerably prominent, the application based around Android SDK appeals to much more attention. Because of technological advancement, mobile terminals have actually evolved into functionally sophisticated devices such as, for instance smart phones and tablets. The Android platform has grown to become one of the most popular operating system alongside millions of new users each and every year. In spite of many studies, no one have presented a comprehensive classification of this particular operating system. In this particular paper we present an impartial review of the Android OS. We summarize the platforms historical past which includes improvements associated with each and every release as well as the systems engineering and project structure.
\end{abstract}

\section{General Terms}

Google Android Mobile Operating System.

\section{Keywords}

Google, Android, Lollipop, Android OS

\section{INTRODUCTION}

Android is a software platform and operating system for mobile phone devices, in accordance with the Linux kernel, and put together by Google and subsequently the Open Handset Alliance. It permits developer team to write and manage program code within the Java language, controlling the product via Google manufactured Java depository library. Android is actually available as open source. Android is an accomplished extensively down-loadable open source software stack for mobile devices that comes with an operating system, middleware and key applications based around Linux and Java. Search engine Google purchased the developer of Android in 2005, as well as Android was launched in 2007. Google launched the Android code as opensource according to the Apache License. Android has numerous programmers, writing applications (apps) all over the world. Before everything else, the developers write their script in Java, immediately after which they download the apps from the third party sites or online stores. In the month of April 2016, 2 Billion apps were available for Android but the approximated number of downloads since December, 2015 was more than 1.25 billion .Android is actually usually the one of the most extremely used mobile operating system which include a market share of $70.85 \%[1]$ and Over $2,200,000[2]$ applications available in Google play store. Android apps have been installed over 10 billion times and cover a vast range of categories coming from games as well as entertainment to financial and business services. Android software development along with the Google Play Market is reasonably open and un-exclusive. This offers developers as well as users more flexibility and freedom simultaneously, but also creates appreciable security challenges. Google Play, earlier known as Android Market, is a digital application distribution platform for Android as well as online electronics store developed and managed by Google. The service makes it possible for users to browse as well as download music, books, magazines and catalogs as well as applications circulated through Google.

\section{ANDROID ARCHITECTURE}

Google Android system is a Linux-based system that uses the software stack architecture design patterns [3-4] .As shown in Figure 1, the Android architecture consists of four layers: Linux kernel, Libraries and Android runtime, Application framework and Applications ${ }^{[5-6]}$.Each layer regarding the lower encapsulation, while supplying call interface towards upper. Similar to other platforms, applications can be obtained from a dedicated place called Google Play ${ }^{[7]}$. The Android system architecture shown in Fig. 1.consists of five layers: Linux kernel, libraries, Android runtime, application framework and applications ${ }^{[8]}$.

\subsection{APPLICATIONS}

Android app will likely be delivered having a group of core applications incorporating client, SMS program, calendar, roadmaps, browser, contacts, as well as others. All these application programs are designed in object-oriented programming language Java.

\subsection{Application Framework}

The developer is actually permitted to access all of the API framework of the core programs. The application framework makes easier the recycling of its components. Any other app can easily release its functional components and all sorts of other apps have access to and make use of this component (but need to proceed with the security of the framework). Just like the users tends to be in a position to substitute the program components with this particular reuse mechanism.

\subsection{Libraries And Google Android Runtime}

The library is split straight into two components: Android Runtime as well as Android Library. Android Runtime actually consists of a Java Core Library and Dalvik[9] virtual machine. The Core Library provides Java core library with many functions. Dalvik virtual machine is actually register virtual machine and tends to make some specific enhancements for mobile device. Google android system library actually supports the application framework, it is normally a significant link connecting somewhere between application framework as well as Linux Kernel[10]. This system library is actually developed in $\mathrm{C}$ or $\mathrm{C}++$ language. These types of libraries could possibly be applied because of the variety of elements within the Google android system. 
They provide service for the developers through the application framework.

\subsection{Linux Kernel}

The kernel system service supplied by Google android inner nuclear layer is dependent on Linux 2.6 kernel; Operations such as internal storage, process management, internet communications protocol, bottom-drive and other core service are typically based on Linux kernel.

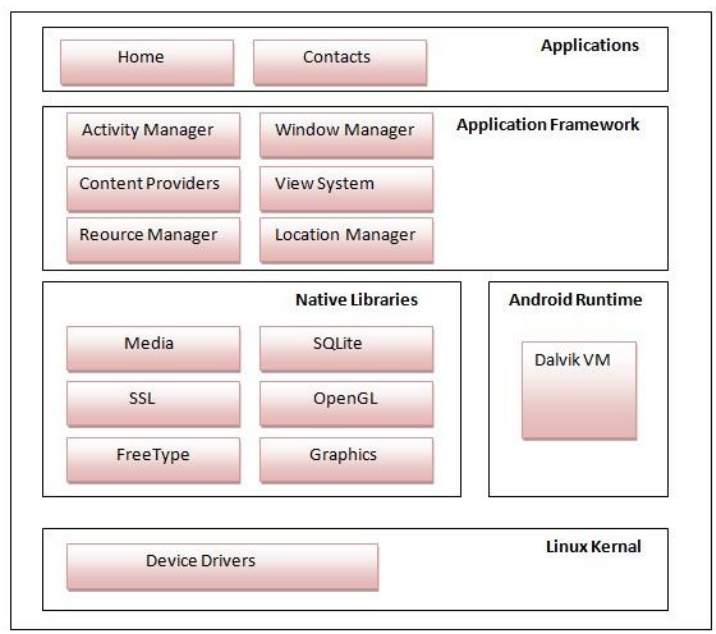

Fig 1: Android Operating System Architecture

\section{VERSION HISTORY}

Android is modernizing day by day since its release. These types of upgrades to the base operating system primarily concentrating on dealing with bugs together with incorporating additional features to provide additional accustomed environment. In many instances each and every latest version of the Android operating system is actually matured under a code name according to a dessert item. Several Of Android Operating System versions are

\subsection{Cupcake}

Technologically Android 1.5 wasn't the first version, but versions earlier don't appear to have gained any codenames. Stories were explained it absolutely was supposed to be version 1.2, but Google decided to transform it into a significant revision and made it 1.5 instead. Among the numerous adjustment with Cupcake, third-party pc style keyboard as well as Widgets were enabled and phone could possibly upload straight to YouTube and Picasa. The corporation codenamed the actual version "cupcake," which is exactly how the trend of dessert names began. A cupcake is really a compact, individually-sized cake baked within a cupshaped mold. Most commonly it is served with frosting on top

\subsection{Donut}

Android V1.6, codenamed "Donut," was actually released in September 2009. It resolved restart flaws in the OS, refurbished graphic as well as digital video features (i.e. camera interface), and introduced much better search integration. Moreover it incorporated support for much larger screen sizes and is the first version to offer Google's turn-byturn navigation characteristic. A donut is a compact ringshaped fried cake. The ring is made of productive, light dough and deep-fried. A variety of sweet coatings can be added.

\subsection{Eclair}

Google android 2.0 was released in October 2009, by having a bug fix version (2.0.1) being released in December 2009. Android 2.1 was released in January of 2010. The majority of people contemplate them a single release. Additional features include Bluetooth 2.1 support, flash and digital zoom for the digital camera, multi-touch influence, live wallpapers, and more. Eclairs are generally characterized as oblong cream puffs.

\subsection{Froyo}

Google android 2.2 primarily improved speed by adopting the JavaScript "just-in-time" compiler engine from Google's browser, Chrome. Additionally, it improved browser support with the addition of animated GIF support and Flash 10.1 plug-in support, in conjunction with Universal serial bus tethering as well as Wi-Fi Hotspot functionality (for individuals with supporting hardware).

\subsection{Gingerbread}

Gingerbread was technologically released in December 2010. On December 6th, 2010, Google officially proclaimed the very first mobile phone with Android OS 2.3 Gingerbread. The mobile phone was the Nexus $\mathrm{S}$, which Google codeveloped alongside Samsung. The device ended up being originally only accessible for T-Mobile, but was subsequently made for the Sprint and AT\&T as well. Gingerbread is compatible with SIP internet calling, NFC wireless transaction capability (if hardware is present), more than one digital camera, and gyroscopes and other sensors (barometers, gravimeters, as well as others tend to be possible). Additionally it incorporates a download manager, some tweaks to allow usage on Tablets, along with other system level tweaks for programmers.

\subsection{Honeycomb}

Honeycomb was introduced in February 2011, and was aggressively followed by 3.1 and 3.2 in July and August of 2011. Google published plenty of previews as well as highlights on Honeycomb. Honeycomb was developed for tablets.

\subsection{Ice Cream Sandwich}

Ice Cream Sandwich was Google's endeavor to synthesize Honeycomb, usually tablet-only platform, with its mobile

platform. Released in October 2011, it highlighted an innovative new design and standard font, as well as the opportunity to monitor and throttle mobile data usage along with other upgrades. Many devices were ponderous to embrace Ice Cream Sandwich.

\subsection{Jelly Bean}

Jelly Bean came out in 2012. Most significant adjustment incorporated "Google Now," an Artificial Intelligence associate that anticipates your requirements and much better, even more interactional notifications. Jelly Bean also permits "voice typing," a built-in speech-to-text engine that doesn't depend upon Internet or data.

\subsection{Kitkat}

Google proclaimed that Android 4.4 is going to be titled KitKat on September 3, 2013. KitKat's parent company, KitKat procured the Google Now feature and took it one step deeper with "Ok Google." Ok Google gives people the ability to access Google Now without even touching their phonesjust vocally expressing the phrase opens up the artificial 
intelligence associate. KitKat additionally introduced Emoji to Google's keyboard.

\subsection{Lollipop}

Google android 5 is referred to as Lollipop, and it highlighted a brand new runtime called ART that eliminate on the older DALVIK runtime (which is nearly based on Sun/Oracle specs). Lollipop also incorporates other UI advancements and has now an outstanding battery-life on some devices

\subsection{Marshmallow}

Android 6: Marshmallow is already out for the Nexus devices and is also believed to be coming soon to all primary devices prior to end of the season, and also to other devices by mid 2016. Marshmallow unveiled a number of modifications that can have considerable influence. App authorization model is now opt-in (grant specific authorization as requested) as opposed to opt-out (all is authorized, then use App Ops to run off particular person permissions). Doze mode enables the device to enter into hibernation when idle, slicing electrical power consumption to virtually nil. Fingerprint sensing unit support is now baked into the OS rather the vendor assistance, and USB C is now completely supported. Subsequently,

Marshmallow allows one to format a micro SD card as well as embrace it as if it really is internal storage space and share the exact same internal security measures level.

\section{ANDROID SECURITY}

The open natures of Google android as well as its large user base have managed to get an appealing as well as worthwhile platform to attack. Common intrusions as well as tool kits throughout the OS can be employed across an extensive number of devices, which means that attackers can perform intrusions to re-use attack vectors. Google did take strategies within the development of the Google android kernel to construct security measures in; the OS is actually sandboxed, controlling detrimental processes from traversing in between applications. Whilst this attempt to eradicate the reasoning behind infection is remarkable in several regards, it ceases to address the issue of infection completely.

Google android is an accomplished victim of its very own success, not simply in the manner it has fascinated malicious attention, but in its very nature. A primary reason this OS was able to succeed in acquiring share of the market so rapidly is that it is open source; it is essentially complimentary for manufacturers to implement. Additionally this has concluded in considerable atomization concerning Google android versions anywhere between devices as well as means that companies have now been unwilling to roll-out advancements, presumptively out of some concern pertaining to traveling requirement for foreseeable future devices.

\section{FEATURES OF ANDROID OPERATING SYSTEM}

\subsection{Storage}

SQLite, a light-weight relational database, is utilized for data storage space purposes

\subsection{Connectivity}

Google android is compatible with online connectivity technological innovation incorporating GSM EDGE, IDEN, CDMA, EVDO, UMTS, Bluetooth, Wifi, LTE, NFC as well as WI MAX.

\subsection{Messaging}

Text Message as well as MMS are generally available different types of messaging, which also include threaded text messaging and Google's Android Cloud to Device Messaging (C2DM) and currently improved version of C2DM, Android Google Cloud Messaging (GCM) is additionally a part of Android Push Messaging service.

\subsection{Multiple language assistance}

Google's Android supports numerous different languages.

\subsection{Web browser}

The internet browser easily obtainable in Android, is founded on the open source Web Kit design engine, coupled alongside Chrome's V8 JavaScript engine. The web browser ratings 100/100 on the Acid3 test on Android 4.0

\subsection{Java support}

Although many Google's Android programs tend to be written in Java, generally there is absolutely no Java Virtual Machine in the working platform as well as Java byte code is certainly not executed. Java classes tend to be compiled into Dalvik executable and run on Dalvik, a specialized virtual machine manufactured particularly for Google's Android as well as optimized for battery-powered mobile phones with minimal memory space as well as CPU. J2ME support can be made available through third party applications.

\subsection{Multi-touch}

Google's Android has native support towards multi-touch which had been initially made accessible in mobile phone models such as the HTC Hero. The characteristic was earlier impaired at the kernel level (potentially to prevent infringing Apple's patents regarding touchscreen display technology at the time). Google offers since released an update for the Nexus One as well as the Motorola Droid which in turn enables multi-touch natively

\subsection{Bluetooth}

Google Android Sustains A2DP, AVRCP, sending files (OPP), being able to access the phone book (PBAP), voice dialing and sending contacts anywhere between phones. Keyboard, computer mouse as well as joystick (HID) support is accessible in Google's Android 3.1+, and in prior versions through manufacturer customizations and third - party applications.

\subsection{Tethering}

Google's Android supports tethering, which makes it possible for a mobile phone to be utilized as wireless/wired Wi-Fi hotspot. Prior to Google's android 2.2 this was fully supported by the 3rd party applications or perhaps manufacturer custom remaking.

\subsection{Screen Capture}

Google's Android supports capturing a screen shot through pressing the electrical power as well as volume-down buttons at the same time. Right before Google's Android 4.0, the only solutions to capturing a screen shot happened to be through manufacturer and additionally third-party customizations or commonly simply by using a PC connection (DDMS developer's tool). These types of alternative techniques are nevertheless available because of the latest Android. 


\section{CONCLUSION}

The extended assistance coming from big corporations, specifically Google, have made Google's Android quite possibly the most significant contestants within the mobile sector. The extensive as well as accessibility to smart phones and tablets make it possible for manufacturers to customize the system in order to suit their demands, such as both hardware as well as software layers. Nevertheless, the inconvenient component of this particular platform, which is certainly fragmentation, still remains. It requires a while for designers and manufacturers to consider an innovative new version of Google's Android to previously released devices available in the industry. Commonly they don't construct an uninterrupted support for almost all. Regardless almost all complications, the release of an innovative new version takes place approximately once a year. The development by itself may perhaps broaden as more computer hardware as well as software companies get working on the project. It really is worth talking about, that the working platform is actually well supported by people outside of the primary Google's Android project.

\section{REFERENCES}

[1] https://www.netmarketshare.com/operating-systemmarket-share .aspx?qprid=8\&qpcustomd $=1$

[2] http://www.statista.com/statistics/276623/number-ofapps-available-in-leading-app-stores/

[3] M. Butler, "Android: Changing the Mobile Landscape", Pervasive Computing, (2011), pp. 4-7.

[4] B. Proffitt, "Open Android-For better and for worse", Spectrum, (2011), pp. 22- 24

[5] A. Shabtai, Y. Fledel and Y. Elovici, "Securing AndroidPowered Mobile Devices Using SELinux", Security \& Privacy, (2010), pp. $36-44$.

[6] X. Zhao and D. Tian, "The Architecture Design of Streaming Media Applications for Android OS", ICSESS,(2012), pp. $280-283$.

[7] Wang C., Duan W. Ma J., Wang C. (2011 ). The Research of Android System Architecture and Application Programming. ICCSNT

[8] Macario G., Torchiano M., Violante M. (2009). An InVehicle Infotainment Software Architecture Based on Google Android.SIES

[9] Wei T.E., Jeng A.B., Lee H.M., Chen C.H., Tien C.W. (2012) Android Privacy. ICMLC

[10] Kundu T.K., Paul K. (2010) Android on Mobile Devices: An Energy Perspective, CIT 